
\title{
25 Research Square \\ The Diagnostic Value of DNA Repair Gene in Breast Cancer Recurrence and Metastasis
}

\section{Yongxin Yang}

Southwest Medical University

Xiabin Li

Southwest Medical University

\section{Liyue Hao}

Southwest Medical University

\section{Deyong Jiang}

Centers for Disease Control and Prevention

\section{Bin Wu}

Southwest Medical University

Tao He

Southwest Medical University

Yan Tang ( $\nabla$ tangyan200310@163.com )

\section{Research}

Keywords: PARP1, XRCC4, ERCC1, Breast cancer

Posted Date: June 25th, 2020

DOI: https://doi.org/10.21203/rs.3.rs-36932/v1

License: (c) (1) This work is licensed under a Creative Commons Attribution 4.0 International License.

Read Full License 


\section{Abstract}

Background: DNA repair genes play a vital role in the treatment of many cancers, and DNA repair genes can be used in breast cancer recurrence and metastasis research. We found that the expression of DNA repair genes in breast cancer patients after recurrence and metastasis is abnormal, however, the clinical predictive significance of DNA repair genes is still elusive.

Methods: The nested case-control method was used in patients with breast cancer recurrence and metastasis after surgery $(n=109)$ and patients without recurrence and metastasis after surgery $(n=109)$. The proteins and mRNA of DNA repair genes were detected by immunohistochemistry and Real-time PCR respectively.

Results: PARP1(OR=1.485, 95\%Cl:1.279 1.725, $P<0.05)$, XRCC4(OR= 1.419, 95\%Cl:1.217 1.656, $P<0.05)$ and ERCC1 $(O R=1.181,95 \% \mathrm{Cl}: 1.032 \sim 1.353, P<0.05)$ were risk factors for postoperative recurrence and metastasis of breast cancer. Therefore, we used the ROC curve to study the optimal critical values of PARP1, XRCC4, and ERCC1, combined with the comprehensive judgment of clinical experience, we can conclude that PARP1 (cutoff value $=6$, Se $=75.23 \%, S p=79.82 \%$ ), XRCC4 (cutoff value $=6, \mathrm{Se}=78.9 \% 0$, $\mathrm{Se}=79.82 \%$ ), ERCC1 (cutoff value $=3, \mathrm{Se}=89.91 \%, \mathrm{Sp}=47.71 \%$ ), suggesting that when the PARP1 score is higher than 6 or the XRCC4 score is higher than 6 or the ERCC1 score is higher than 3 , the risk of recurrence and metastasis will increases $\left(O R_{\mathrm{PARP} 1}=14.941,95 \% \mathrm{Cl}_{\mathrm{PARP} 1}: 4.004 \sim 55.758, P<0.05\right.$; $O R_{\mathrm{XRCC} 4}=16.740,95 \% \mathrm{ClXRC4}_{\mathrm{X}}: 6.433 \sim 43.560, P<0.05 ; O R_{\mathrm{ERCC} 1}=5.285,95 \% \mathrm{Cl}_{\mathrm{ERCC} 1}: 1.843 \sim 15.156, P$ $<0.05)$. Due to PARP1, ERCC1 and XRCC4 belong to a part of DNA repair gene system, and the three proteins are positively correlated by correlation analysis $\left(r_{\mathrm{PARP} 1-\mathrm{ERCC} 1}=0.317 ; r_{\mathrm{PAPR} 1-\mathrm{XRCC} 4}=0.329 ; r_{\text {ERCC1- }}\right.$ $\mathrm{XRCC} 4=0.377$ ). The combined diagnosis of the PARR1,ERCC1 and XRCC4 have greater predictive value for the risk of recurrence and metastasis of breast cancer $(\mathrm{Se}=88.99 \%, \mathrm{Sp}=82.57 \% \otimes O R=50.914,95 \% \mathrm{Cl}$ : $10.918,237.417, P<0.05)$.

Conclusions: The recurrence and metastasis of breast cancer are predictive after PARP1 $>6, \mathrm{XRCC} 4>6$, and ERCC1>3. The combined diagnosis of the three indicators have greater predictive value for the risk of recurrence and metastasis of breast cancer.

\section{Background}

Breast cancer is the most general malignancy in China and either world, and its mortality rate firstly in female malignancy[1]. In recent years, the survival rate of breast cancer has been prominently improved by comprehensive treatment such as surgery and chemotherapy. Nevertheless, approximately one-third of breast cancer patients will present metastases. Recurrence and metastasis are bound up with the prognosis of breast cancer patients and it is also the soprattutto cause of death in breast cancer patients[2]. Studies have found that breast cancer patients' postoperative recurrence and metastasis are related to age, tumor pathological tissue type, clinical analysis, postoperative chemotherapy, and endocrine therapy[3]. At the same time, some people have also studied tolerance to treatment as one of 
the influencing factors. However, some tumor cells can pass activating self DNA repair mechanisms to resistance to DNA damage drugs[4-6]. So some studies have proposed that DNA repair genes have a relationship with the recurrence and metastasis of breast cancer[7].

More and more studies have found that tumor response to chemotherapy drugs is closely related to the regulation of the DNA repair system [8]. Four major DNA repair pathways are currently known: nucleotide excision repair (NER), base excision repair (BER), mismatch repair (MMR), and double strand break repair (DSBR). In cancer, we found that ERCC1, XPA, XRCC1, PARP1, MSH2, MLH1, 53BP1, XRCC4 are closely related to cancer recurrence and metastasis. The ERCC1 and XPA genes in the NER pathway have confirmed that ERCC1 is associated with recurrence and metastasis in ovarian cancer, testicular germ cell tumors and gastric cancer, and high expression of ERCC1 will lead to an increased risk of recurrence and metastasis [9]. BER as one of the DNA repair mechanisms, PARP1 may be one of the major genes involved in tumor cell recurrence and metastasis [10]. In vitro and in vivo studies have suggested that inhibition of PARP1 can reduce tumor cell repair function, thereby enhancing the therapeutic effect of radiotherapy and chemotherapy on tumors $[11,12]$. DSBR is the most common but most severe type of DNA damage in eukaryotic cells, and is mainly repaired in mammals through non-homologous end joining (NHEJ). Xiaoyan Li et al. found that 53BP1 affects breast cancer patients' sensitivity to 5-Fu, it will results poor prognosis [13]. Some scholars have found that the research on DNA repair genes such as MLH1, XRCC4, 53BP1, ERCC1 and XPA in breast cancer is mainly expressed in breast cancer tissues and its clinical significance[14-16]. XRCC1 overexpression can inhibit breast cancer cell proliferation and metastasis[17]. MSH2 mutation may be involved in the occurrence and development of early-onset breast cancer in the family of Lynch syndrome[18]. Among them, PARP1 inhibitors have entered the trial stage of clinical treatment of breast cancer[19]. But no further study of their recurrence and metastasis with breast cancer.

DNA repair requires the role of multiple enzymes and genes. A single gene has a limited role in damage repair. Analyzing only an enzyme or gene is not enough to reflect the complexity of DNA repair. Due to ERCC1, XPA, XRCC1, PARP1, MSH2, MLH1, 53BP1, and XRCC4 are more studied in other cancer. But there are few studies in breast cancer recurrence and metastasis. So in this study, nested case-control study was used to explore the expression levels of major molecules of the DNA repair system ERCC1, XPA, XRCC1, PARP1, MSH2, MLH1, 53BP1, and XRCC4 in patients with recurrent and metastatic breast cancer, in order to provide theoretical support for clinical treatment and prognosis.

\section{Methods}

\subsection{Sample}

The data come from the follow-up cohort of the Cancer Institute of Southwest Medical University. The cohort was collected and followed up in January 2013 at the Department of Breast Medicine, Southwest Medical University Hospital. Cancer patients have collected approximately 1360 cases. Recurrence and metastasis cases and controls selected in this study were collected from this cohort. Patients with 
recurrence and metastasis during the follow-up period were included in the recurrence and metastasis case group. At the same time, the control group(non-recurrence) was selected according to the 1: 1 pairing principle in this cohort $(n=109$, the matching condition was age \pm 3 years, the operation time was consistent, and the treatment plan was consistent). The control group(non-recurrence) was surviving patients in the cohort, and no recurrence and metastasis occurred. Finally, 109 cases and 109 controls were included in January 2018. The pathological data used in this study were from the Department of Pathology, Affiliated Hospital of Southwest Medical University. The data collected included clinical data, pathological data, and treatment options, as well as paraffin specimens from patients with breast cancer. After preliminary diagnosis of breast cancer patients in the affiliated hospital of Southwest Medical University, materials were obtained from the Department of Pathology.

Ethical issues: (1) Patients with informed consent to participate. (2) This research program has been reviewed by the ethics committee of the unit, and it is considered that it meets the relevant ethical requirements of clinical research and agrees to the research plan. Application acceptance number: XNYD2018001.

\subsection{Detection of DNA repair genes ERCC1, XPA, XRCC1, PARP1, MSH2, MLH1, 53BP1 and XRCC4 in paraffin-embedded tissues of breast cancer patients by Real-time PCR}

Total RNA were extracted using the RNeasy FFPE Kit (QIAGEN, shanghai, China), according to manufacturer's instructions. cDNA was reversely transcribed using the PrimeScript ${ }^{\text {TM }}$ RT reagent Kit with gDNA Eraser (TaKaRa, Dalian, Liaoning, China).Gene expression was quantified by SYBRß Premix Ex Tap II (TaKaRa, Dalian, Liaoning, China) and performed in a real-time thermal cycler qTOWER 2.0/2.2 (Analytik Jena, Germany) Relative gene expression was calculated using the $2-\Delta \Delta C T$ method and the results were normalized with $\beta$-actin as an internal control. The primer sequences are shown in Table 1.

Table.1 The primers used for PCR 


\begin{tabular}{|c|c|}
\hline Gene & Primer sequences \\
\hline \multirow[t]{2}{*}{ 53BP1 } & sense primer 5'-CCAGACTCCACCAGACGAACA-3' \\
\hline & Anti-sense 5'-ACCACTTGGCTACAACACGGA-3' \\
\hline \multirow[t]{2}{*}{ ERCC1 } & sense primer 5'-TATGAGCAGAAACCAGCGGAC-3' \\
\hline & Anti-sense 5'-GCTCGTGCAGGACATCAAACA - 3' \\
\hline \multirow[t]{2}{*}{ MLH1 } & sense primer 5'-TGAGGAAGGGAACCTGATTGG - 3' \\
\hline & Anti-sense 5'-CCGGATGGAATAGAACATAGCG - 3' \\
\hline \multirow[t]{2}{*}{ XRCC4 } & sense primer 5'-TCTGTTCTGAAATGACTGCTGACC-3' \\
\hline & Anti-sense 5'- GGTGCAATATCAGTGACATCAAGAC-3' \\
\hline \multirow[t]{2}{*}{ MSH2 } & sense primer 5'-GGAACTTCTACCTACGATGGATTTG-3' \\
\hline & Anti-sense 5'-TCAGTGGTGAGTGCTGTGACATG-3' \\
\hline \multirow[t]{2}{*}{ XRCC1 } & sense primer 5'-TCGAGGACTATATGAGTGACCGG-3' \\
\hline & Anti-sense 5'-ACGAACGAATGCCAGGGAG-3' \\
\hline \multirow[t]{2}{*}{ PARP1 } & sense primer 5'-CAGAAGCCGAAACTCTT-3' \\
\hline & Anti-sense 5'-GATGCCAAATCACCAGGT-3' \\
\hline \multirow[t]{2}{*}{ XPA } & sense primer 5'-TGTTTTGCCTCTGTTTTGGTT-3' \\
\hline & Anti-sense 5'-GTAATATGCGAAGAATGTGGG-3' \\
\hline \multirow[t]{2}{*}{$\beta$-actin } & sense primer 5'-CCACGAAACTACCTTCAACTCC-3' \\
\hline & Anti-sense 5'-GTGATCTCCTTCTGCATCCTGT-3' \\
\hline
\end{tabular}

\subsection{Immunohistochemical detection of DNA repair gene protein expression in paraffin-embedded tissues of breast cancer patients}

Paraffin sections $(3 \mu \mathrm{m})$ were dried, deparaffined, and rehydrated in graded alcohol to water. Heatmediated antigen retrieval was performed using pressure cooker treatment for 10 min in EDTA buffer ( $\mathrm{pH}$ 9.0). The slides were incubated for $120 \mathrm{~min}$ at $25^{\circ} \mathrm{C}$ with primary mouse anti-human monoclonal antibodies to ERCC1, XPA, XRCC1, PARP1, MSH2, MLH1, 53BP1 and XRCC4 (Dako, DK). After washing, the sections were incubated with the second antibody (Envision, HRP rabbit/mouse, Dako, DK) for $30 \mathrm{~min}$ at $25^{\circ} \mathrm{C}$. Negative controls were obtained by omitting the primary antibody. The slides were visualized by DAB.

Expression of 8 DNA repair protein was determined in the nucleus of tumor cells. Five high-power fields (200x) were randomly selected. The extent of the staining was categorized into five semi-quantitative classes based on the percentages of positive tumor cells: $0,<5 \%$ positive cells; $1,6-25 \%$ positive cells; 2 , 
$26-50 \%$ positive cells; $3,51-75 \%$ positive cells; and $4,>75 \%$ positive cells. Staining intensity was scored as 0 , negative; 1 , weak; 2 , moderate; and 3 , intensive. Multiplication of the intensity and the percentage scores gave rise to the final staining score[20].

\subsection{Statistical analysis}

All data were analyzed using SPSS 22.0 statistical software and MedCalc software, and bilateral $P$ values below 0.05 were considered statistically significant. The correlation between DNA repair genes and breast cancer recurrence and metastasis was analyzed by $\chi^{2}$ test, rank sum test, cox risk model and other statistical methods.

\section{Results}

\subsection{The protein expression of DNA repair genes}

Immunohistochemical staining results (Fig. 1) shows that: DNA repair gene protein positive expression mainly in the cytoplasm, repair gene is highly expressed in the transfer of recurrence group in the breast tissue. The MSH2, MLH1, PARP1, XRCC1, XRCC4, 53BP1, ERCC1, XPA of the recurrence and metastasis group were higher than those of the control group(non recurrence and metastasis group) $(P<0.05)$, we can concluded that all of them are related to the prognosis of recurrence and metastasis of breast cancer.

\subsection{The mRNA expression of DNA repair genes}

Fig. 2 shows the comparison of the expression of DNA repair gene mRNA in breast cancer patients in the recurrence and metastasis group and the control group(non recurrence and metastasis group). The mRNA expressions of MSH2, MLH1, PARP1, XRCC1, XRCC4, 53BP1, ERCC1 and XPA in breast cancer recurrence and metastasis group were higher than those in control group(non recurrence and metastasis group) $(P<0.05)$.

\subsection{Clinicopathologic feature of breast cancer patients}

The PR index of the recurrence and metastasis group was lower than that of the control group(non recurrence and metastasis group $)(P<0.05)$. P53, WHO Grade and Lymph node metastasis of the recurrence and metastasis group was higher than that of the control group(non recurrence and metastasis group) $(P<0.05)$. There was no significant difference in Age, ER, HER2, E-cad, Ki67, TNBC, Infiltration and Tumor diameter between the two groups $(P>0.05)$, as shown in Table 2 .

Table.2 Clinicopathologic feature of breast cancer patients [n(\%)] 


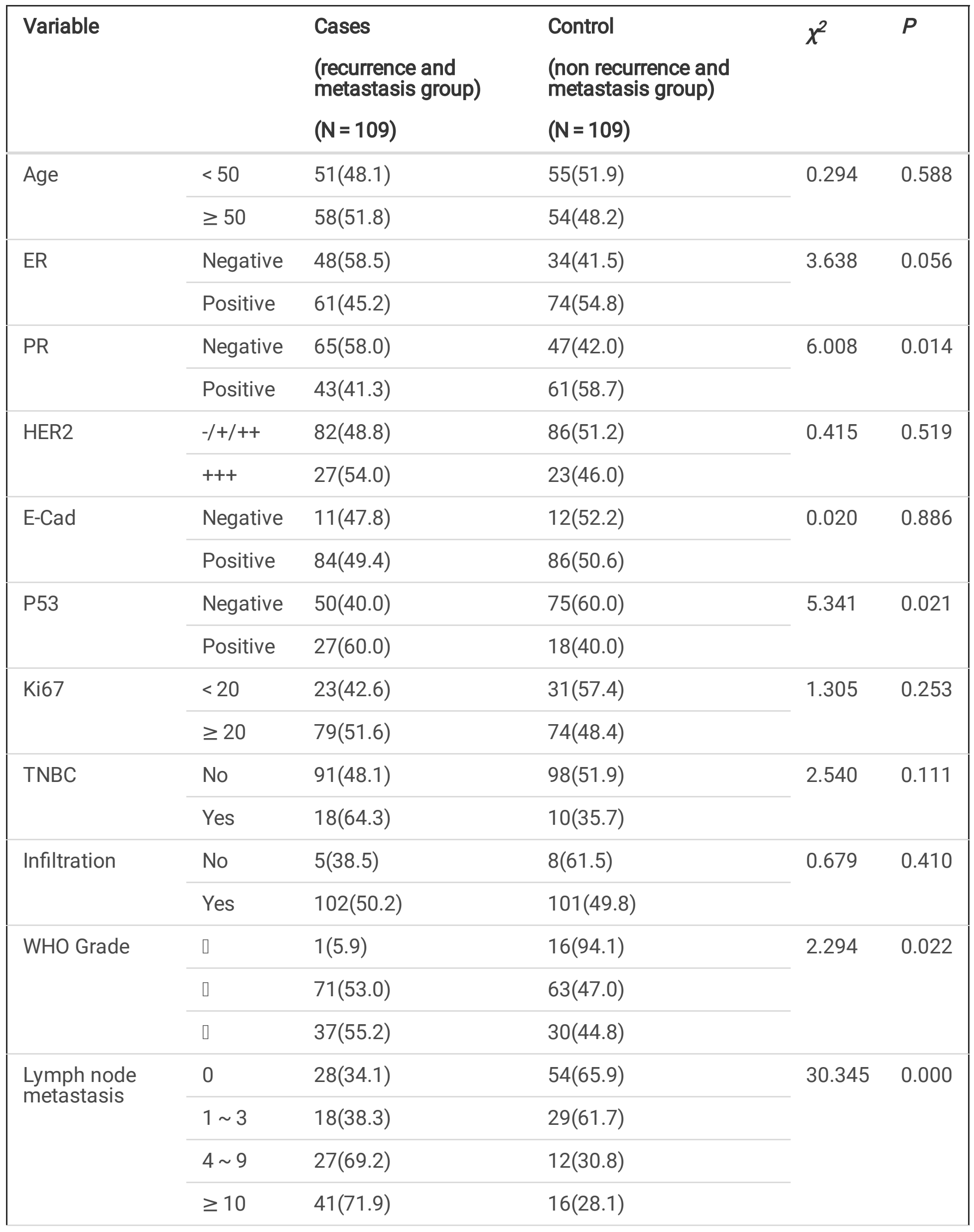




\begin{tabular}{|llllll|}
\hline Variable & Cases & $\begin{array}{l}\text { Control } \\
\text { (recurrence and } \\
\text { metastasis group) } \\
(\mathbf{N}=109)\end{array}$ & $\begin{array}{l}\text { (non recurrence and } \\
\text { metastasis group) } \\
(\mathbf{N}=109)\end{array}$ & $\chi^{2}$ & $P$ \\
\hline Tumor diameter & $<2 \mathrm{~cm}$ & $28(43.8)$ & $36(56.2)$ & \multirow{2}{*}{1.416} & 0.234 \\
\cline { 2 - 5 } & $\geq 2 \mathrm{~cm}$ & $81(52.6)$ & $73(47.4)$ & & \\
\hline
\end{tabular}

\subsection{Binary Logistic Regression Analysis}

To reduce confounding bias, at the protein expression level and the mRNA expression level, respectively, binary logistic regression analysis was performed on variables related to prognosis in univariate analysis. The results showed that at the protein level, PARP1 $(O R=1.485,95 \% \mathrm{Cl} .1 .279 \sim 1.725, P<0.05), \mathrm{XRCC} 1(O R$ $=1.293,95 \% \mathrm{Cl}: 1.083 \sim 1.543, P<0.05), \mathrm{XRCC} 4(O R=1.419,95 \% \mathrm{Cl}: 1.217 \sim 1.656, P<0.05)$ and ERCC1 $(O R=$ $1.181,95 \% \mathrm{Cl}: 1.032 \sim 1.353, P<0.05)$ were risk factors for postoperative recurrence and metastasis of breast cancer. The results of mRNA levels showed that the PARP1(OR=1.770,95\% Cl:1.376 2.276, $P<$ $0.05), \operatorname{XRCC} 4(O R=1.264,95 \% \mathrm{Cl}: 1.055 \sim 1.515, P<0.05)$ and $\operatorname{ERCC1}(O R=1.489,95 \% \mathrm{Cl}: 1.182 \sim 1.876, P$ $<0.05)$ were risk factors for postoperative recurrence and metastasis of breast cancer. For details, see Tables 3 and 4.

Table 3

Binary logistic regression of protein expression

\begin{tabular}{|llllll|}
\hline Variable & $\boldsymbol{B}$ & S.E. & Wald & $\boldsymbol{P}$ & OR(95\%CI) \\
\hline PR & -0.845 & 0.492 & 2.950 & 0.086 & $0.430(0.164,1.127)$ \\
\hline PARP1 & 0.396 & 0.076 & 26.891 & 0.000 & $1.485(1.279,1.725)$ \\
\hline XRCC1 & 0.257 & 0.090 & 8.082 & 0.004 & $1.293(1.083,1.543)$ \\
\hline XRCC4 & 0.350 & 0.079 & 19.887 & 0.000 & $1.419(1.217,1.656)$ \\
\hline ERCC1 & 0.167 & 0.069 & 5.822 & 0.016 & $1.181(1.032,1.353)$ \\
\hline Lymph node metastasis & & & & & \\
\hline 0 & - & - & - & - & Reference \\
\hline $1 \sim 3$ & 0.193 & 0.649 & 0.089 & 0.766 & $1.213(0.340,4.327)$ \\
\hline $4 \sim 9$ & 2.098 & 0.746 & 7.918 & 0.005 & $8.150(1.890,35.142)$ \\
\hline$\geq 10$ & 2.704 & 0.672 & 16.197 & 0.000 & $14.941(4.004,55.758)$ \\
\hline
\end{tabular}


Table 4

Binary logistic regression of mRNA expression

\begin{tabular}{|llllll|}
\hline Variable & $\boldsymbol{B}$ & S.E. & Wald & $\boldsymbol{P}$ & OR(95\%CI) \\
\hline PR & -1.107 & 0.497 & 4.956 & 0.026 & $0.331(0.125,0.876)$ \\
\hline PARP1 & 0.571 & 0.128 & 19.744 & 0.000 & $1.770(1.376,2.276)$ \\
\hline XRCC4 & 0.234 & 0.092 & 6.456 & 0.011 & $1.264(1.055,1.515)$ \\
\hline ERCC1 & 0.398 & 0.118 & 11.430 & 0.001 & $1.489(1.182,1.876)$ \\
\hline XPA & -0.744 & 0.265 & 7.866 & 0.005 & $0.475(0.282,0.799)$ \\
\hline Lymph node metastasis & & & & & \\
\hline 0 & - & - & - & - & Reference \\
\hline $1 \sim 3$ & 0.564 & 0.643 & 0.771 & 0.380 & $1.758(0.499,6.195)$ \\
\hline $4 \sim 9$ & 1.442 & 0.720 & 4.010 & 0.045 & $4.228(1.031,17.337)$ \\
\hline$\geq 10$ & 2.352 & 0.622 & 14.280 & 0.000 & $10.509(3.102,35.598)$ \\
\hline
\end{tabular}

\subsection{Diagnostic value of DNA repair genes}

In the previous study, we found that the protein expression of PARP1, XRCC4 and ERCC1 is related to the recurrence and metastasis of breast cancer $(P<0.05)$. However, the IHC score is a continuous variable and there is no accurate cut-off value for diagnosis. In order to further understand the role of PARP1, ERCC1 and XRCC4 in the prognosis of breast cancer recurrence and metastasis. Therefore, we used the ROC curve to study the optimal critical values of PARP1, XRCC4, and ERCC1, combined with the comprehensive judgment of clinical experience, we can conclude that PARP1 (cutoff value $=6, \mathrm{Se}=$ $75.23 \%, \mathrm{Sp}=79.82 \%$ ), $\mathrm{XRCC} 4$ (cutoff value $=6, \mathrm{Se}=78.9 \% 0, \mathrm{Se}=79.82 \%$ ), ERCC1 (cutoff value $=3, \mathrm{Se}=$ $89.91 \%, \mathrm{Sp}=47.71 \%$ ), suggesting that when the PARP1 score is higher than 6 or the XRCC4 score is higher than 6 or the ERCC1 score is higher than 3, the risk of recurrence and metastasis will increases. As shown in Fig. 3 and Table 5. Based on the cutoff value predicted by ROC curve and clinical experience, PARP1, XRCC4 and ERCC1 were divided into the high expression group and the low expression group according to the cutoff value, and the effects of PARP1, XRCC4 and ERCC1 on breast cancer recurrence and metastasis were verified again. Among them, the risk of recurrence and metastasis in the PARP1 high expression group was 14.235 times that of the low expression group $(O R=14.235,95 \%$ Cl.5.59 36.245, $P<0.05)$. The risk of recurrence and metastasis in the XRCC4 high expression group was 16.740 times that of the low expression group $(O R=16.740,95 \% \mathrm{Cl}$ : $6.433 \sim 43.560, P<0.05)$. The risk of recurrence and metastasis in patients with ERCC1 high expression group was 5.285 times that of the low expression group $(O R=5.285,95 \% \mathrm{Cl}: 1.843 \sim 15.156, P<0.05)$. The risk of recurrence and metastasis in patients 
with lymph node metastasis $\geq 10$ was 9.175 times that without lymph node metastasis $(O R=9.175$, 95\% Cl:2.842 29.625, $P<0.05)$. As shown in Table 6.

Table 5

The best diagnostic value of PARP1, XRCC4 and ERCC1

\begin{tabular}{|c|c|c|c|c|}
\hline Indicator & $\begin{array}{l}\text { Cut-off } \\
\text { Value }\end{array}$ & Sensitivity(\%) & Specificity(\%) & Youden index \\
\hline \multirow[t]{3}{*}{ PARP1 } & 4 & 91.74 & 52.29 & 0.4403 \\
\hline & 6 & 75.23 & 79.82 & 0.5505 \\
\hline & 8 & 61.47 & 89.91 & 0.5138 \\
\hline \multirow[t]{3}{*}{ XRCC4 } & 4 & 96.33 & 50.46 & 0.4679 \\
\hline & 6 & 78.90 & 79.82 & 0.5872 \\
\hline & 8 & 56.88 & 86.24 & 0.4312 \\
\hline \multirow[t]{3}{*}{ ERCC1 } & 2 & 91.74 & 44.04 & 0.3578 \\
\hline & 3 & 89.91 & 47.71 & 0.3761 \\
\hline & 4 & 74.31 & 61.47 & 0.3578 \\
\hline
\end{tabular}

Table 6

Binary logistic regression of protein high expression and low expression in postoperative recurrence and metastasis of breast cancer

\begin{tabular}{|llllll|}
\hline Variable & B & S.E. & Wald & $P$ & OR(95\%Cl) \\
\hline PARP1 & 2.656 & 0.477 & 31.015 & 0.000 & $14.235(5.590,36.245)$ \\
\hline XRCC4 & 2.818 & 0.488 & 33.351 & 0.000 & $16.740(6.433,43.560)$ \\
\hline ERCC1 & 1.665 & 0.538 & 9.593 & 0.002 & $5.285(1.843,15.156)$ \\
\hline Lymph node metastasis & & & & & \\
\hline 0 & - & - & - & - & Reference \\
\hline $1 \sim 3$ & 0.133 & 0.571 & 0.054 & 0.816 & $1.142(0.373,3.496)$ \\
\hline $4 \sim 9$ & 2.423 & 0.731 & 10.975 & 0.001 & $11.278(2.690,47.290)$ \\
\hline$\geq 10$ & 2.216 & 0.598 & 13.737 & 0.000 & $9.175(2.842,29.625)$ \\
\hline
\end{tabular}




\subsection{The correlate and joint diagnostic value on between PARP1, XRCC4, ERCC1}

Due to PARP1, ERCC1 and XRCC4 belong to a part of DNA repair gene system, and the three proteins are positively correlated by correlation analysis $\left(r_{\mathrm{PARP} 1-\mathrm{ERCC} 1}=0.317 ; r_{\mathrm{PAPR} 1-\mathrm{XRCC} 4}=0.329\right.$;

$\left.r_{\text {ERCC1-XRCC4 }}=0.377\right)$. See Table 7. These results indicate that there is an internal connection between these three proteins, and there is a certain synergy between them. So we combined PARP1, ERCC1 and XRCC4 to detect the prognosis of breast cancer. Joint diagnostic criteria: the high expression of a single indicator is judged as high, while the three indicators are simultaneously low and judged to be low.(Se = $88.99 \%, \mathrm{Sp}=82.57 \% ; O R=50.914,95 \%$ Cl. 10.918, 237.417, $P<0.05)$.See Fig. 4 and Table 8.

Table 7

The correlation coefficient of PARP1, XRCC4 and ERCC1

\begin{tabular}{|llll|}
\hline Variables & $\mathbf{N}$ & $\boldsymbol{r}$ & $\boldsymbol{P}$ \\
\hline PARP1-XRCC4 & 218 & 0.329 & 0.000 \\
\hline PARP1-ERCC1 & 218 & 0.317 & 0.000 \\
\hline XRCC4-ERCC1 & 218 & 0.377 & 0.000 \\
\hline *:Spearman. & & & \\
\hline
\end{tabular}

Table 8

The Youden index and OR of combine detection

\begin{tabular}{|llllll|}
\hline Joint indicator & Sensitivity(\%) & Specificity(\%) & $\begin{array}{l}\text { Youden } \\
\text { index }\end{array}$ & OR(95\%CI) & $P$ \\
\hline $\begin{array}{l}\text { PARP1 + XRCC4 } \\
\text { +ERCC1 }\end{array}$ & 88.99 & 82.57 & 0.7156 & $50.914(10.918,237.417)$ & 0.000 \\
\hline
\end{tabular}

\section{Discussion}

Chemotherapy is one of the most important treatments for breast cancer after operation. At present, the survival rate of patients has been effectively improved by referring to ER, PR, HER-2, Ki67, TNBC and other indicators. However, the study found that there are still about $30 \%$ recurrence and metastasis rates [21]. It shows that the formulation of treatment plans based on the above pathological indicators may be incomplete, and there are other indicators for guiding treatment that can be excavated. In addition, there is still rise the survival rate of patients for adjustment in the formulation of treatment plans. Nowadays, more and more studies have found that the recurrence and metastasis of tumors are closely related to the DNA repair regulatory system related to drug resistance[22-24].

At present, not a few DNA repair genes, such as PARP1, XRCC1, 53BP1, ERCC1, have been found to be associated with the prognosis of breast cancer[25,26]. PARP1 promotes the expression of HIF-1a by 
activating nuclear factor- $\mathrm{KB}(\mathrm{NF}-\mathrm{kB})$ and promotes the polarization of macrophages $\mathrm{M} 2$, leading to the up-regulation of tumor-related macrophages (TAMs), such as tumor necrosis factor-a (TNFa) and IL-6, thus promoting the proliferation, invasion and metastasis of tumor cells, promoting the formation of tumor microvessels and microlymphatics[27]. And ERCC1 proteins can form heterodimers with DNA repair enzyme deficiency complementary gene (XPF) and perform functions by splicing at the 5 'end of the damaged DNA single strand. Overexpression of ERCC1 proteins can lead to rapid repair of damaged DNA stagnating in $\mathrm{G} 2 / \mathrm{M}$, leading to resistance to cisplatin chemotherapeutics[28]. In this study, nested case-control single factor analysis showed that protein levels of PARP1, XRCC1, 53BP1, ERCC1 recurrence and metastasis group was higher than control group. We further included that PARP1 (OR $=1.485,95 \%$ Cl:1.279-1.725), ERCC1 (OR=1.181, 95\% Cl:1.032-1.353) as independent factors for recurrence and metastasis of breast cancer after operation. The results are similar to those of Sunada, S. et al[29]. It shows that XRCC1 and 53BP1 become ineffective factors after excluding other confounding factors, while the high expression of PARP1 and ERCC1 in the course of chemotherapy will destroy the susceptibility of breast cancer cells, it can be make breast cancer cells less susceptible to chemotherapy, leading to resistance of breast cancer cells and even recurrence and metastasis of chemotherapy failure, which is affecting breast cancer. The independent prognostic factors of metastatic patients are not different from the existing studies.

In addition to the above genes related to breast cancer resistance[30], the prognosis-related DNA repair genes MSH2, MLH1, XRCC4 and XPA[31-33], which are frequently studied in the fields of colorectal cancer, lung cancer, prostate cancer and esophageal cancer, were also included in the study and analysis. Univariate analysis showed that MSH2, MLH1, XRCC4 and XPA in patients with recurrence and metastasis were higher than those without recurrence and metastasis. In binary logistic regression, only XRCC4 (OR $=1.419,95 \% \mathrm{Cl}: 1.217 \sim 1.656)$ was a risk factor for recurrence and metastasis of breast cancer, suggesting that XRCC4 was also associated with recurrence and metastasis of breast cancer after operation. The results of high expression of XRCC4 in esophageal and colorectal cancer suggest poor prognosis. It is possible that XRCC4 itself could form dimers and interact with BRCT domain (BRCA1-terminal, BRCT) at the $\mathrm{C}$ end of Ligase IV protein through its a spiral stem ring structure in the central region. Damage repair defects lead to accumulation of genetic damage, and then increase the susceptibility of the body to tumors, thereby increasing the risk of recurrence and metastasis of breast cancer patients.

However, the direct use of IHC score to analyze the recurrence and metastasis of breast cancer after surgery is of little significance. In order to further understand the role of PARP1, ERCC1 and XRCC4 in predicting the prognosis, metastasis and recurrence of breast cancer, we also studied the best cut-off value of PARP1, ERCC1 and XRCC4. The results showed that PARP1 (Cut-off Value $=6, \mathrm{Se}=75.23, \mathrm{Sp}=$ 79.82), ERCC1 (Cut-off Value $=3, \mathrm{Se}=89.91, \mathrm{Sp}=47.71$ ), XRCC4 (Cut-off Value $=6, \mathrm{Se}=78.90, \mathrm{Se}=$ 79.82), suggesting that the risk of recurrence and metastasis increased at the scores of PARP1, ERCC1 and XRCC4 were higher than 6, 3, 6, respectively(PARP1: $O R=14.235,95 \% \mathrm{Cl} .5 .590 \sim 36.245$; ERCC1: $O R=$ 16.740, 95\% Cl:6.433 43.560; XRCC4: OR= 5.285, 95\% Cl:1.843 15.156), the risk of breast cancer recurrence and metastasis will increases. The sensitivity of PARP1, ERCC1 and XRCC4 single detection is 
between $75.23 \% \sim 89.91 \%$, the specificity is between $47.71 \% \sim 79.82 \%$, the Youden index is between $0.3761 \sim 0.5872$, the sensitivity is qualified, but the specificity and Youden index are low. It indicates that the diagnostic value of individual tumor markers in the prognosis of breast cancer needs to be further improved. Due to PARP1, ERCC1 and XRCC4 belong to a part of DNA repair gene system, and the three proteins are positively correlated by correlation analysis $\left(r_{\mathrm{PARP} 1-\mathrm{ERCC} 1}=0.317 ; r_{\mathrm{PAPR} 1-\mathrm{XRCC} 4}=0.329\right.$; $r_{\text {ERCC1-XRCC4 }}=0.377$ ). These results suggest that there is an internal link among the three proteins and there is a certain synergy among them. So we combined PARP1, ERCC1 and XRCC4 to detect the prognosis of breast cancer. Joint diagnostic criteria: the high expression of a single indicator is judged as high, while the three indicators are simultaneously low and judged to be low. The results showed that after using the joint test, the specificity of diagnosis increased from $47.71-82.57 \%$. The Youden index increased from 0.3761 to 0.7156 . Sensitivity only decreased from $89.91-88.99 \%$, a slight decrease, but the magnitude is very small. And in the binary logistic regression of breast cancer prognosis, the odds ratio of the combined indicators is as high as 50.914 ( $\mathrm{OR}=50.914,95 \% \mathrm{Cl}: 10.918 \sim 237.417$ ). It can be seen that the combined detection of three DNA repair proteins has higher clinical diagnostic value than the single determination. It has been pointed out that PARP1 promotes the expression of HIF-1a by activating nuclear factor $\mathrm{KB}(\mathrm{NF}-\mathrm{kB})$ and promotes the polarization of macrophages $\mathrm{M} 2$, leading to the up-regulation of tumor-related macrophages (TAMs), such as tumor necrosis factor alpha (TNFa) and IL6 , thus promoting the proliferation, invasion and metastasis of tumor cells, promoting the formation of tumor microvessels and microlymphatics[34]. Overexpression of ERCC1 also up-regulates TNF-a, while XRCC4 in NHEJ pathway activates DNA-dependent protein kinase complex (DNA-PK), which is essential for the adhesion of monocytes to TNF-a treated endothelial cells. Other studies have found that in the process of tumorigenesis and development, most patients are accompanied by chronic inflammation, and the chronic protraction of inflammation affects the condition of tumors, leading to recurrence and metastasis of tumors[35]. Tumor necrosis factor- $a$ (TNFa) is closely related to the occurrence of cancer. The secretion of TNF-a cytokines in tumor microenvironment can accelerate the growth and spread of cancer cells. At the same time, it can make cancer cells bypass the impact of the immune system, promote the EMT process of cells, and cause recurrence and distant metastasis of cancer[36]. These studies suggest that PARP1, ERCC1 and XRCC4 may act together on TNF- $\alpha$ to produce recurrence and metastasis of breast cancer. It can be seen that the combined detection of the three DNA repair proteins has higher clinical diagnostic value than the single determination, which is helpful for the early diagnosis of breast cancer prognosis.

\section{Conclusions}

PARP1 $>6$, XRCC4 $>6$ and ERCC1 $>3$ can predict the recurrence and metastasis of breast cancer. In addition, the combined diagnosis of PARP1,XRCC4 and ERCC1 has great predictive value for the risk of breast cancer recurrence and metastasis. However, the mechanism of the effect of PARP1,XRCC4 and ERCC1 on the recurrence and metastasis of breast cancer remains unclear, which needs further study.

\section{Abbreviations}


ERCC1: DNA excision repair cross complementing 1; XPA: Xeroderma pigmentosum group A; XRCC1: Xray repair cross-complementing 1; PARP1:Poly ADP-ribose polymerase-1; MSH2: MutS homolog 2; MLH1: MutL homolog 1; 53BP1: p53 binding protein; XRCC4: X-ray repair cross-complementing 4; ER: Estrogen receptor; PR: Progesterone receptor; HER2: humanepidermalgrowthfactorreceptor-2; TNBC: Triple negative breast cancer; TAMs: Tumor-related macrophages; TNF-a: Tumor necrosis factor-a; DNA-PK: DNAdependent protein kinase complex; EMT: Epithelial - mesenchymal transition; NF-kB: Nuclear factor-kappa B

\section{Declarations}

\section{Acknowledgements}

We would like to thank all the patients for their contribution in this study.

\section{Authors' contributions}

$\mathrm{Y}-\mathrm{T}$ and $\mathrm{T}-\mathrm{H}$ designed, guided the study; XB-L and $\mathrm{LY}-\mathrm{H}$ performed the experiments; $\mathrm{YX}-\mathrm{Y}$ analyzed the data and wrote the paper; DY-J and B-W collected the samples. The authors read and approved the final manuscript.

\section{Funding}

This work was supported by grants from the Sichuan Science and Technology Plan Project (2015sz0115) and the Luzhou Science and Technology Plan Project (2013LZLY-J37).

\section{Availability of data and materials}

The data and materials of this study are available from the corresponding authors for reasonable requests.

\section{Ethics approval and consent to participate}

Patients with informed consent to participate. This research program has been reviewed by the ethics committee of the unit, and it is considered that it meets the relevant ethical requirements of clinical research and agrees to the research plan. Application acceptance number: XNYD2018001.

\section{Consent for publication}

All authors agree to submit the article for publication. 


\section{Competing interests}

The authors declare that they have no conflicts of interest.

\section{Referrence}

1. Siegel, R. L., K. D. Miller, and A. Jemal. Cancer statistics, 2018. CA Cancer J Clin 2018;68(1):7-30.

2. Redig, A. J., and S. S. McAllister. Breast cancer as a systemic disease: a view of metastasis. J Intern Med 2013;274(2):113-26.

3. Haviland, J. S. et al. The UK Standardisation of Breast Radiotherapy (START) trials of radiotherapy hypofractionation for treatment of early breast cancer: 10-year follow-up results of two randomised controlled trials. Lancet Oncol 2013;14(11):1086-1094.

4. Genois, M. M., et al. DNA repair pathways in trypanosomatids: from DNA repair to drug resistance. Microbiol Mol Biol Rev 2014;78(1):40-73.

5. Srivastava, M., and S. C. Raghavan. DNA double-strand break repair inhibitors as cancer therapeutics. Chem Biol 2015;22(1):17-29.

6. Wang, S., et al. DNA Repair Genes ERCC1 and BRCA1 Expression in Non-Small Cell Lung Cancer Chemotherapy Drug Resistance. Med Sci Monit 2016;22:1999-2005.

7. Teply, B. A., and E. S. Antonarakis. Treatment strategies for DNA repair-deficient prostate cancer. Expert Rev Clin Pharmacol 2017;10(8):889-898.

8. Ma, R., et al. Mechanisms involved in breast cancer liver metastasis. J Transl Med 2015;13:64.

9. Mendoza, J., et al. Association between ERCC1 and XPA expression and polymorphisms and the response to cisplatin in testicular germ cell tumours. $\mathrm{Br} J$ Cancer 2013;109(1):68-75.

10. Zhu, Q., et al. Autoimmune response to PARP and BRCA1/BRCA2 in cancer. Oncotarget 2015;6(13):11575-84.

11. Oei, A. L., et al. Sensitizing thermochemotherapy with a PARP1-inhibitor. Oncotarget 2017;8(10):16303-16312.

12. Lin, L., et al. The clinicopathological significance of miR-149 and PARP-2 in hepatocellular carcinoma and their roles in chemo/radiotherapy. Tumour Biol 2016;37(9):12339-12346.

13. Li, X., et al. 53BP1 sensitizes breast cancer cells to 5-fluorouracil. PLoS One 2013;8(9):e74928.

14. Allen-Brady Kristina,Cannon-Albright Lisa A,Neuhausen Susan L et al. A role for XRCC4 in age at diagnosis and breast cancer risk.[J] .Cancer Epidemiol. Biomarkers Prev., 2006, 15: 1306-10.

15. Li Xiaoyan,Xu Bing,Moran Meena S et al. 53BP1 functions as a tumor suppressor in breast cancer via the inhibition of NF-KB through miR-146a.[J] .Carcinogenesis, 2012, 33: 2593-600.

16. Palomba Grazia,Atzori Francesco,Budroni Mario et al. ERCC1 polymorphisms as prognostic markers in T4 breast cancer patients treated with platinum-based chemotherapy.[J] .J Transl Med, 2014, 12: 272. 
17. Ali Reem,Al-Kawaz Abdulbaqi,Toss Michael S et al. In SituTargeting PARP1 in XRCC1-Deficient Sporadic Invasive Breast Cancer or Preinvasive Ductal Carcinoma Induces Synthetic Lethality and Chemoprevention.[J] .Cancer Res., 2018, 78: 6818-6827.

18. Harkness Elaine F,Barrow Emma,Newton Katy et al. Lynch syndrome caused by MLH1 mutations is associated with an increased risk of breast cancer: a cohort study.[J] .J. Med. Genet., 2015, 52: 553-6.

19. Efimova Elena V,Mauceri Helena J,Golden Daniel W et al. Poly(ADP-ribose) polymerase inhibitor induces accelerated senescence in irradiated breast cancer cells and tumors.[J] .Cancer Res., 2010, 70: 6277-82.

20. Hao, L., et al. Recombination of CXCR4, VEGF, and MMP-9 predicting lymph node metastasis in human breast cancer. Cancer Lett 2007;253(1):34-42.

21. Dittmer, J. Mechanisms governing metastatic dormancy in breast cancer. Semin Cancer Biol 2017;44:72-82.

22. Liu, C., et al. Personalised pathway analysis reveals association between DNA repair pathway dysregulation and chromosomal instability in sporadic breast cancer. Mol Oncol 2016;10(1):179-93.

23. Williams, D. T., and C. J. Staples. Approaches for Identifying Novel Targets in Precision Medicine: Lessons from DNA Repair. Adv Exp Med Biol 2017;1007:1-16.

24. Vareslija, D., et al. Adaptation to Al Therapy in Breast Cancer Can Induce Dynamic Alterations in ER Activity Resulting in Estrogen-Independent Metastatic Tumors. Clin Cancer Res 2016;22(11):2765-77.

25. Hassan, S., et al. Pathway-Enriched Gene Signature Associated with 53BP1 Response to PARP Inhibition in Triple-Negative Breast Cancer. Mol Cancer Ther 2017;16(12):2892-2901.

26. Zhang, Z., et al. Triptolide interferes with XRCC1/PARP1-mediated DNA repair and confers sensitization of triple-negative breast cancer cells to cisplatin. Biomed Pharmacother 2019;109:15411546.

27. Raman, V., et al. Lippia origanoides extract induces cell cycle arrest and apoptosis and suppresses NF-kappaB signaling in triple-negative breast cancer cells. Int J Oncol 2017;51(6):1801-1808.

28. Guyon-Debast, A., et al. The XPF-ERCC1 Complex Is Essential for Genome Stability and Is Involved in the Mechanism of Gene Targeting in Physcomitrella patens. Front Plant Sci 2019;10:588.

29. Sunada, S., A. Nakanishi, and Y. Miki. Crosstalk of DNA double-strand break repair pathways in poly(ADP-ribose) polymerase inhibitor treatment of breast cancer susceptibility gene 1/2-mutated cancer. Cancer Sci 2018;109(4):893-899.

30. Calaf, G. M., et al. Molecular aspects of breast cancer resistance to drugs (Review). Int J Oncol 2015;47(2):437-45.

31. Gerodimos, C. A., et al. Effects of DNA end configuration on XRCC4-DNA ligase IV and its stimulation of Artemis activity. J Biol Chem 2017;292(34):13914-13924.

32. Kim, J., et al. DNA Mismatch Repair Protein Immunohistochemistry and MLH1 Promotor Methylation Testing for Practical Molecular Classification and the Prediction of Prognosis in Endometrial Cancer. Cancers (Basel) 2018;10(9). 
33. Ma, X. J., et al. Mitotic regulator Nlp interacts with XPA/ERCC1 complexes and regulates nucleotide excision repair (NER) in response to UV radiation. Cancer Lett 2016;373(2):214-21.

34. Awasthee, N., et al. Anti-cancer activities of Bharangin against breast cancer: Evidence for the role of NF-kappaB and IncRNAs. Biochim Biophys Acta Gen Subj 2018;1862(12):2738-2749.

35. DeMichele, A., D. Yee, and L. Esserman. Mechanisms of Resistance to Neoadjuvant Chemotherapy in Breast Cancer. N Engl J Med 2017;377(23):2287-2289.

36. Dominguez, C., J. M. David, and C. Palena. Epithelial-mesenchymal transition and inflammation at the site of the primary tumor. Semin Cancer Biol 2017;47:177-184.

\section{Figures}

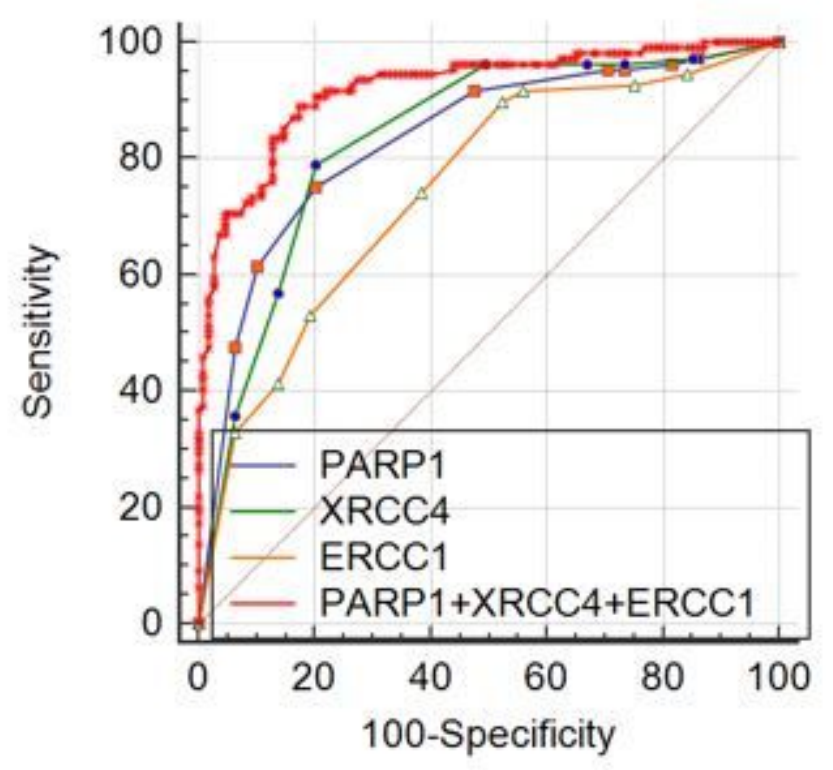

Figure 1

ROC curve of PARP1, XRCC4 and ERCC1 combined to diagnose breast cancer recurrence and metastasis 


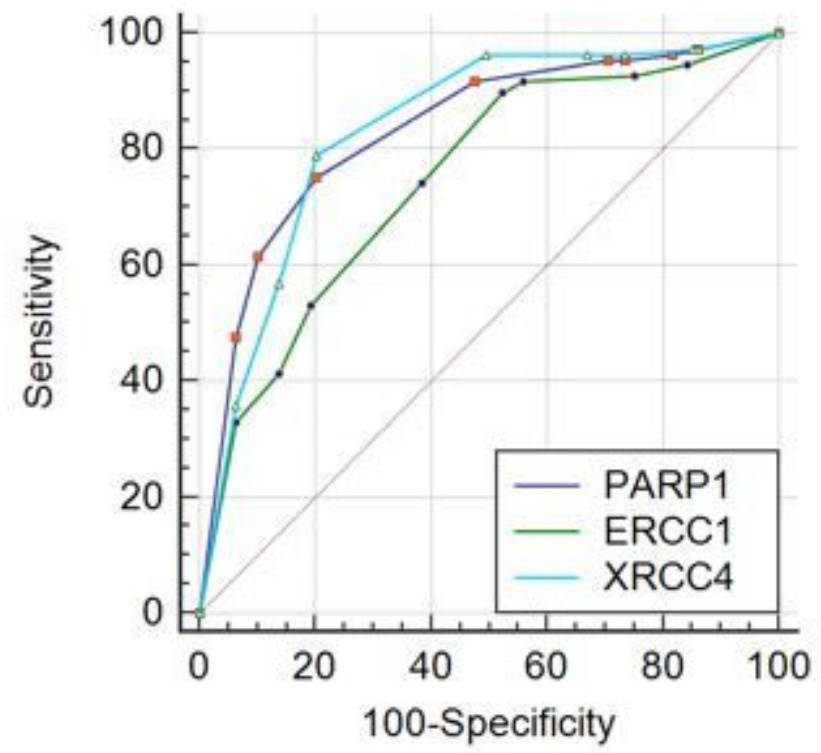

Figure 2

The diagnose ROC curve of PARP1, XRCC4 and ERCC1

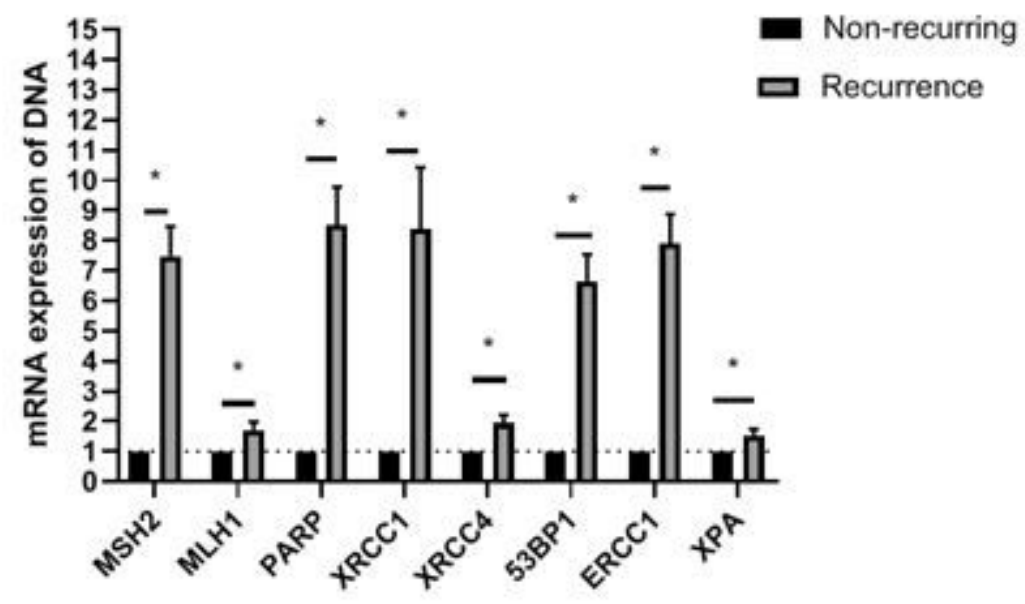

Figure 3

Shows the effect of breast cancer recurrence and metastasis on the mRNA expression of DNA repair gene. Data are described as Mean (SD), $n=109$. Statistical differences are expressed as: ${ }^{*} P<0.05$. 

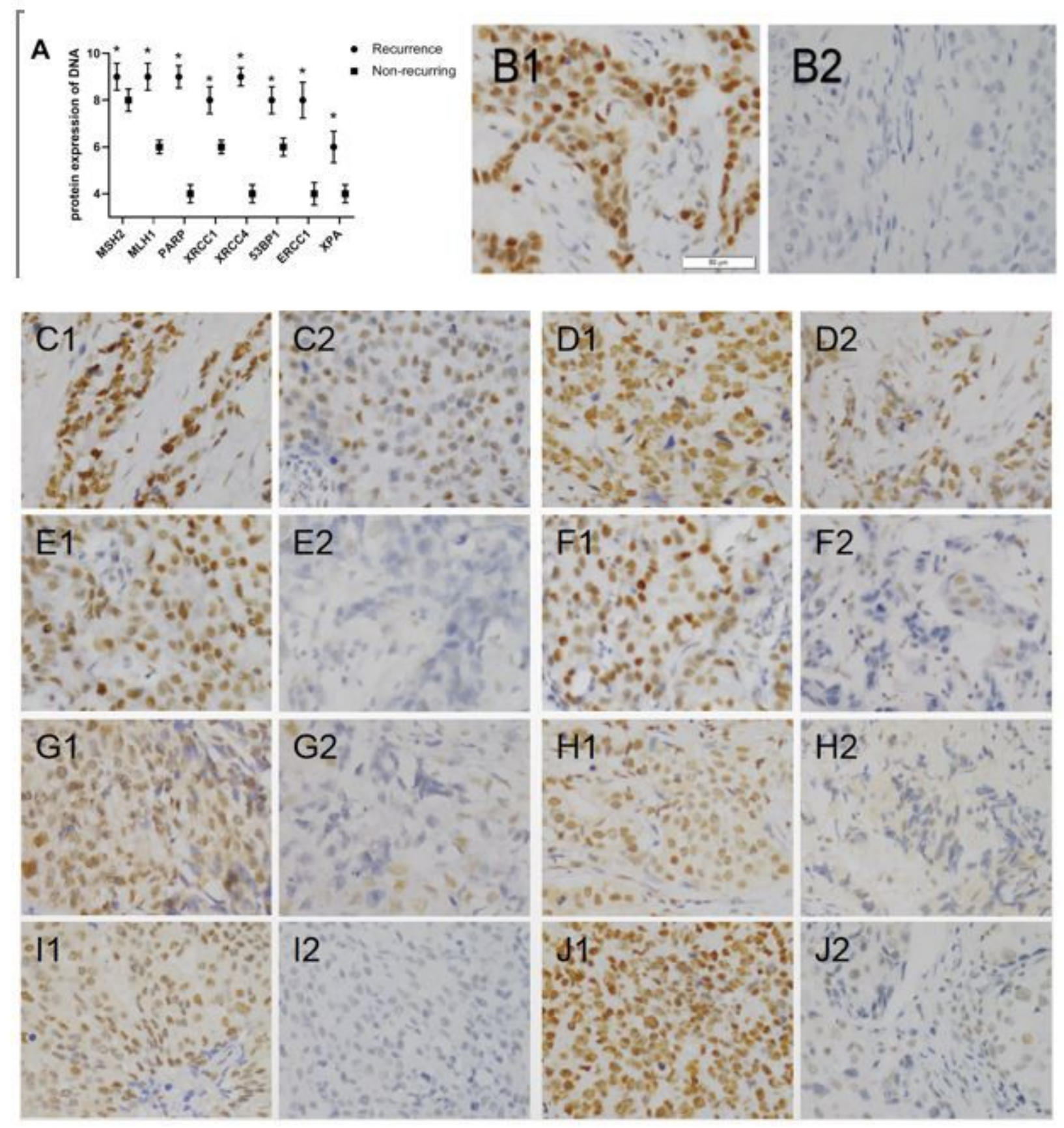

\section{Figure 4}

Shows the effect of breast cancer recurrence and metastasis on the protein expression of DNA repair gene as shown in Figure 1A. Data are described as Median (IQR), $N=109$. Statistical differences are expressed as: ${ }^{*} P<0.05$. Strong expression of immunohistochemical positive controls compared to negative controls (B). Immunohistochemistry (IHC) detection of DNA repair genes MSH2 (C), MLH1 (D), PARP1 $(E)$, XRCC1 $(F)$, XRCC4 $(G), 53 B P 1(H)$, ERCC1 (I), XPA (J) in paraffin tissues of patients with recurrence and metastasis breast cancer ( 1 for the recurrence and metastasis group, 2 for the control group(non recurrence and metastasis group); original magnification $\times 400$ ). 